\title{
Business Intelligence in Maternity Care
}

\author{
Eliana Pereira \\ Computer Science and \\ Technology Center (CCTC) \\ University of Minho \\ Braga, Portugal \\ Manuel Filipe Santos \\ Algoritmi Research Center \\ University of Minho \\ Guimarães, Portugal \\ mfs@dsi.uminho.pt
}

a60196@alunos.uminho.pt a58549@alunos.uminho.pt

\author{
Andreia Brandão \\ Computer Science and \\ Technology Center (CCTC) \\ University of Minho \\ Braga, Portugal \\ José Machado \\ Computer Science and \\ Technology Center (CCTC) \\ University of Minho \\ Braga, Portugal \\ jmac@di.uminho.pt
}

\author{
Carlos Filipe Portela \\ Algoritmi Research Center \\ University of Minho \\ Guimarães, Portugal \\ cfp@dsi.uminho.pt
}

\author{
António Abelha \\ Computer Science and \\ Technology Center (CCTC) \\ University of Minho \\ Braga, Portugal \\ abelha@di.uminho.pt
}

\begin{abstract}
The emergency services are usually pressured to make quick decisions with incomplete information on most cases, and this situation has a significant impact on healthcare as well on increasing medical errors. On the other hand, there has been an increase of the Electronic Health at Maternity Care. The combination of these two factors allows the construction of a Decision Support System specific for Maternity Care Unit using Business Intelligence technology. This solution is supported by a Data Warehouse, that uses the dimensional structure snowflake and makes the modeling of the maternity care database. With this solution it is intended to turn possible a clinical evidence-based practice, allowing for real time medical decision making with pervasive and interoperable characteristics. This paper presents the architecture, KPIs and benefits of Business Intelligence solution for the real context. This platform has several modules of clinical importance. The Obstetric Gynecological Emergency and the Voluntary Interruption of Pregnancy modules are object of study. This solution has an innovative contribution to the medical and scientific community studying the problem in Maternity area.
\end{abstract}

\section{Categories and Subject Descriptors}

H.4 [Information Systems Applications]: Miscellaneous; D.2.8 [Software Engineering]: Metrics - complexity measures, performance measures

Permission to make digital or hard copies of part or all of this work for personal or classroom use is granted without fee provided that copies are not made or distributed for profit or commercial advantage and that copies bear this notice and the full citation on the first page. Copyrights for components of this work owned by others than ACM must be honored. Abstracting with credit is permitted. To copy otherwise, to republish, to post on servers or to redistribute to lists, requires prior specific permission and/or a fee. IDEAS '14'14, July 07 - 09 2014, Porto, Portugal

Copyright (C) 2014 ACM 978-1-4503-2627-8/14/-7 $\$ 15.00$ http://dx.doi.org/10.1145/2628194.2628248.

\section{General Terms}

Theory

\section{Keywords}

Business Intelligence; Data Mining: Triage; Emergency Department; Intelligent Decision Support Systems; Manchester Triage System; Pervasive; Real-time; Interoperability.

\section{INTRODUCTION}

The Information and Communication Technology (ICT) have a great potential for increasing the quality and efficiency of the healthcare system allowing information sharing, communication, collaboration and coordination between care providers. With this, it increases the presence of Electronic Health Records (EHR) in healthcare institutions [6, 9]. Based on this scenario, the concept of Business Intelligence (BI) has attracted enormous attention between health professionals and professionals of Information Technologies (IT) for Health as to its applicability in the EHR $[2,9]$.

In urgencies of Maternidade Júlio Dinis (MJD), the EHR platform is supported by the Agency for Integration, Archive and Diffusion of Medical Information (AIDA) since 2010 [4]. The fact that the EHR consists of many records on patients makes it a good database to application of BI technology [9, 2]. Due to the large amount of data, it is difficult for a clinician to have an idea of the type of patients that are received and their characteristics. Therefore and in order to support the decision process, arises the development of a comprehensive BI platform for maternity enabling real-time (acquisition, processing and provision of information), a review of Key Perform Indicators (KPI) for the care of the patients of Gynecologists and Obstetrics(GO). This platform in addition to data relating to triage should also be added to other indicators of episodes recorded for a maternity care such as Voluntary Interruption of Pregnancy (VIP) and forecasts of Data Mining (DM).

That said, this article is focused on presenting an architecture of global BI that can be used in hospitals, taking as a case study to MJD. It is planned apply BI and DM techniques in order to develop a platform where is possible 
generate useful KPI clinical and management for healthcare professionals in the context of the Module of GO Emergency and in the Module of VIP.

Beyond the introduction, the article includes five sections. The first one is related to the background and relative work done in the MJD. Topics such as interoperability, general terms of the process BI used in this project and the process of DM are addressed. The third section outlines the BI system that's being implemented in MJD and some of the indicators to get at the end. Finally, some discussions and conclusions are made and future work is suggested.

\section{BACKGROUND AND RELATED WORK}

\subsection{Interoperability}

In the Centro Hospitalar do Porto (CHP), the interoperability between Information Systems is guaranteed by AIDA. It is an agency that is based on the use of intelligent agents to enable communication between different systems in the CHP. This multi-agent system enables the standardization of clinical systems and even beyond the medical and administrative complexity of the different sources of hospital information. Thus allows the sending and receiving of hospital information, management, storage and response to requests for information from third parties [8,6]. All Medical Information Systems are connected through AIDA, for example, the SAPE (Nursing Support System) and SONHO (clinical information system) $[8,6]$.

\subsection{Maternity Care}

Maternity is an intuition of health that usually occupies the care of gynecologists and obstetrics specialties. In general is done supporting pregnant during pregnancy, since the beginning of pregnancy until delivery consequently neonatal stage. Thus in this type of institutions are professionals (doctors and nurses) specialize in GO in order to meet the medical needs of these patients. In the case of CHP, MJD is the institution that deals with this type of service. The MJD support pregnant patients, non-pregnant women, puerperal disorders, has to consult Voluntary interruption of pregnancy (VIP), and allows the examination of cardiotocography (CTG) is done [4].

\subsection{Business Intelligence and Knowledge Dis- covery in Databases}

The definition of Business Intelligence varies amongst researchers. Normally BI system may be defined as an integrated set of tools, technologies and programmed products that are used to collect, integrate, analyze and make data available [10]. BI transforms information into knowledge and has the capability of putting the right information into the hands of the right user at the right time to support the decision-making process $[10,2]$. In the case of BI, DW is the foundation for business intelligence. The principal architectures development of DW is: Top-Down approach and Bottom-Up approach $[5,1,7]$. In the case of this application the of architectures Bottom-Up approach and Kimball's approach will be flowed.

Data Mining represents a phase of the Knowledge Discovery in Databases process (KDD) and consists in finding patterns or relationships that may exist in the data stored in data repositories. This process includes five stages and aims at obtaining knowledge in order to support the process of decision making. The steps are :Selection; Transformation; Data Mining; Interpretation/Evaluation. There are many methodologies of KDD. One example of this is the Cross Industry Standard Process for Data Mining (CRISP-DM), that will be flowed in this project too.

\section{BUSINESS INTELLIGENCE SOLUTION}

Following the architecture of Kimball described in section 2.3 was drawn BI project for Maternity Care. This project was framed having in consideration the case study: MJD.

\subsection{Problem Definition and Benefits of BI Plat- form for MJD}

In MJD patient records since they are admitted in the maternity ward until they are given the high clinic are recorded and supported by AIDA. AIDA supports the EHR. However in MJD does not exist a decision support system able to use the data that is stored in databases and makes it into useful knowledge that can be used as clinical decision support in real-time, enabling the medical clinic practice system evidence-based [4].

Both in the case of GO emergency as in the case of VIP it appears that the data are stored in EHR. This information is stored in databases, but never return to be used for knowledge extraction, or when used, may be very time-consuming and costly techniques to extract knowledge. One example of that is the development of clinical indicators using rudimentary tools such as worksheets for generate graph or reports, as it has been used in recent years for the case of VIP.

In this case the idea of implementing a BI platform in the VIP module is a follow up to facilitate the work of health professionals, who were responsible for obtaining reports, which should contain indicators related to information recorded during the proceedings. These reports are held annually and are used for evaluation of MJD in the VIP module [3].

Thus, one of the main motivations for developing this BI platform is the ability to optimize and automate the entire process of obtaining indicators as well as the creation of new knowledge so that it can be made available at the time of decision. In addition, this platform still allows to give usability to the high amount of data recorded in the database and that are not used.

In the case of GO module emergency does not exist a process of knowledge extraction. So this platform in this module will be a novelty and an asset to the clinical practice, the doctors that will certainly allow a much safer clinical practice, avoiding wasted time and fewer medical errors.

In addition to these specific situations the platform at the global level will allow an organization of distributed information, extracting only those that may be relevant to motherhood, and build scenarios to help health professionals in decision-making. Also allow greater autonomy and flexibility of the users when it comes to creating reports, possibility one quick and simple analysis of clinical data.

\subsection{BI Architecture}

After defining the problem, according to the architecture 
described in the section 2.3, the next step is to plan the process. In this case the process begins with the selection of the information needed to generate the indicators. In this specific case the information is in the EHR and SAPE. How can been seen the data used are stored in different databases being interconnected by AIDA. Depending on the variables collected and the targets defined, the data will be automatically validated and prepared according the processing and transformation rules. Then the data will be loaded into the DW for multidimensional model previously designed using a schema. This process is called ETL (extract, transform and load). $[5,1,7]$.

In a perfunctory manner was initially collected (extracted) information. This case is appealed to data bases of SAPE and the EHR. In the manufacturing procedures were used to eliminate null, repeated data and transform information. In the loading phase it was used procedures to load/update information for the DW. These procedures are predefined and scheduled tasks in order to automate the process. At a later stage it was chosen an Open source BI tool for construction of On-Line Analytical Process (OLAP) Cube, and thus visualize the information/indicators requested in MJD through reports, graphs, dashboards,...etc, as seen in figure 1 , where architecture and the tools used for the construction of this BI platform is presented.

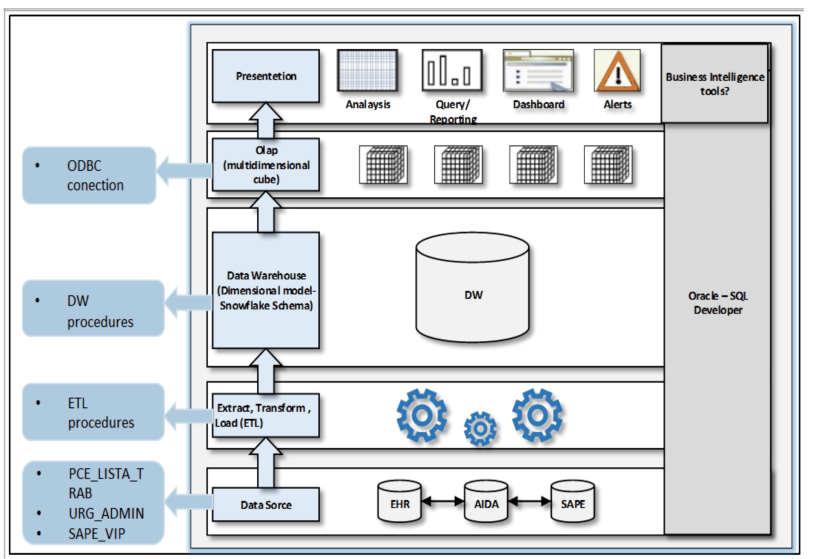

Figure 1: BI architecture.

\subsection{Key Perform Indicators}

This is a first selection of indicators required by health professionals. However, the BI system is prepared to present new indicators as needed.

In GO Emergency module the indicators that are under development are: Activity of doctors between a certain time periods; Daily Activity;Professional High times; Doctors high clinical; Evolution hourly of the high clinical in emergency obstetric module; Flow of patients arriving in the MJD;

This module also indicators over obstetric nature will be developed such as: Number of births per year, number of eutocic deliveries per year, number of obstructed deliveries (cesarean) per year, number of obstructed births (forceps) per year, number of births by skilled nurses, number of live births and stillborn every year, among others.

On the other hand, the usefulness of implementing a BI platform in the module in VIP is the extraction of knowledge from information recorded in patients from SAPE as obtain indicators of activity, process and results, allowing to have an overall idea of the functioning of MJD of this module.

Some of the indicators to be achieved with the application of BI in the VIP are: characterization of the user group by age; characterization of the set of users in relation to employment; gestational age at the time of the first dose of medication (mifepristone); Characterization of the user group for the number of pregnancies; characterization of the user group for the number of children; Characterization of the user group for the number of VIP previous experiences; Characterization of the user group with previous experience of that VIP and resort to MJD for the second time; Contraception early in the process; Main reasons for failures related to the pill; Contraceptive methods used at the end of the process; Characterization of the user group for the achievement of process (MJD or domicile); number of failures VIP according to the number of weeks of pregnancy the patient; among others.

Furthermore, with the implementation of a BI tool in MJD still intends to make a prediction of which women who require monitoring of nursing in the administration of the second dose of drugs and which are able to do it in the home, with based on some associated variables. This prediction is a process of exploiting large amounts of data in order to find consistent patterns to detect systematic relationships between the variables. This process is called data mining [3]. With data mining, it is expected to predict whether the patient VIP host a second procedure at home or with the accompaniment of a nurse and provide upon certain characteristics of the patients, the VIP was achieved or not, among other predictions.

In the GO using DM techniques too, for induce models to predict the waiting time for patient be answered after triage.

\subsection{BI Application}

The BI application is a web platform with pervasive characteristics, which allows it to be accessed anytime and anywhere, provided to who has access privileges [12]. In BI application are represented all indicators that are intended and also other indicators that can be created by users, using the OLAP. Each indicator is associated with a form of representation, such as tables, bar charts, pie charts, diagrams, among others. As can be seen in the figure $2 \mathrm{~A}$, it is as an example of an indicator represented in a bar graph showing the characterization of the user who performed a VIP for ages.

Regarding OLAP technology, this enables end-users to make $a d-h o c$ analysis on data with multiple dimensions, thus providing the information and knowledge necessary for better decision making. With this tool, various operations can be performed, in particular, the roll-up, drill-down, slice and dice and pivot $[7,11]$. As can be seen in the figure $2 \mathrm{~B}$, there is an example of an OLAP cube with specialties, medical appointments and time dimensions. This cube allows the analysis of the number of appointments by specialty at a given time.

\section{DISCUSSION}

There are several key benefits for integrating the BI tech- 


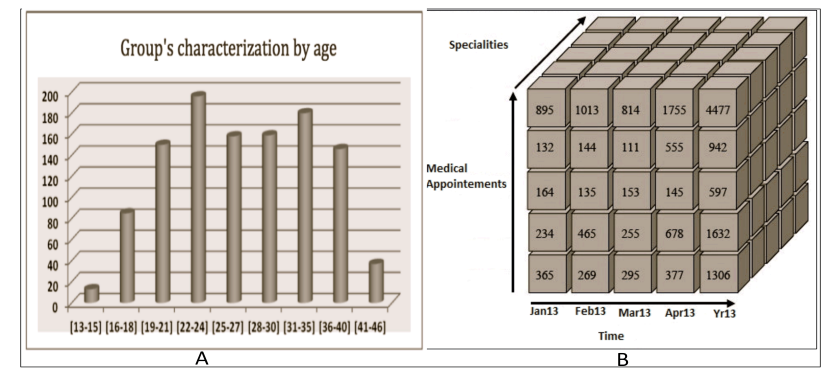

Figure 2: A-Group's characterization by age represented in a bar graph [3]. B-Representation of an example of multidimensional cube for OLAP analysis [7]

nology in MJD. The benefits of the BI to MJD technology can be primarily identified in the form of the increased autonomy and flexibility of users, when it comes to creating reports, quick and simple analyses, improved decision support and operational efficiency, as well as a range of new analytical functions.

In the scientific level this platform will bring many advantages, because in addition to being a pioneer project in the area of BI to maternity care is characterized by specific indicators generated. Taking into account the results obtained and accreditation by health professionals, this platform could provide a starting point for the improvement of these modules. It may also be useful for the research and development of new modules of interest not only to the specialities of obstetrics and gynecologists, as well as for other specialities. Thus, this project could be to the scientific community a starting point for the development of BI platforms in this medical field.

Specifically the usefulness of implementing a BI platform in the GO emergency module in MJD is the extraction of knowledge from data of patients registered in AIDA. The use of these indicators allow a way to have a global idea of the functioning of MJD in this module. Otherwise, the implementation of a BI platform in the module of VIP in MJD would be very advantageous, inasmuch as, for example, allow the interpretation of existing data logs for nursing; indicators give rise to translate the overall operation of this module; would provide improvement strategies continuing in care, based on the results obtained; and disclose the activity carried out in this area.

\section{CONCLUSION AND FUTURE WORK}

This paper presented and explored the importance of integrating BI technology in the Maternity Care. This BI platform it is been in deployment using a real case: MJD and will thus obtain indicators such as daily patient records and clinical activity of doctors during a certain period of time in the emergency module, as well as the characterization of pregnant women who use the VIP module by age, number of pregnancies, among others. The indicators mentioned in this article are just some of the most popular with health professionals. However, this platform also allows to obtain additional indicators that are the target of interest.

Also a growing need to increase the implementation of EHR, it is necessary to apply DM technology to extract quality rules and inference data from the information stored in the electronic records, so as to provide support for realtime decisions doctors and health professionals.

Throughout this study, health professionals identified the lack of a BI system integrated to MJD, because this will increase the autonomy and flexibility to users when it comes to creating reports; allows quick and simple analysis, there are savings time, greater decision support, maximizing evidencebased practice.

The implementation of a BI platform in Maternity, in GO and VIP units, is quite innovative, in that it is the first time that BI technologies are applied in these areas. As already mentioned this platform is undergoing tests.

\section{ACKNOWLEDGEMENTS}

"This work is funded by National Funds through the FCT - Fundação para a Ciência e a Tecnologia (Portuguese Foundation for Science and Technology) within project PEstOE/EEI/UI0752/2014 and PEst-OE/EEI/UI0319/2014. "

\section{REFERENCES}

[1] S. Alcântara. Business Intelligence (BI) como Auxilíar à Gestão do Negócio. Master's thesis, Faculdade de Tecnologias da Zona Leste, São Paulo, 2010.

[2] W. Bonney. Applicability of Business Intelligence in Electronic Health Record. Procedia - Social and Behavioral Sciences, 73:257-262, Feb. 2013.

[3] A. Borges and C. Valente. Acompanhamento de enfermagem na interrupção da gravidez por opção da mulher. 2012.

[4] A. Cabral, A. Abelha, M. Salazar, C. Quintas, F. Portela, J. Machado, J. Neves, and M. Santos. Knowledge Acquisition Process for Intelligent Decision Support in Critical Health Care. IGI Global Book, 2013.

[5] S. Chaudhuri, U. Dayal, and V. Narasayya. An overview of Business Intelligence Technology. Communications of the ACM, 54(8):88, Aug. 2011.

[6] J. Machado, V. Alves, A. Abelha, and J. Neves. Ambient intelligence via multiagent systems in medical arena. International Journal of Engineering Intelligent Systems, Special issue on Decision Support Systems, 15(3):167-173, 2007.

[7] O. Oliveira. Extração de Conhecimento nas Listas de Espera para Consulta e Cirurgia. Master's thesis, Universidade do Minho, 2012.

[8] H. Peixoto, M. Santos, A. Abelha, and J. Machado. Intelligence in interoperability with aida. Springer, Lecture Notes in Computer Science, 7661, 2012.

[9] F. Portela, M. Vilas-Boas, M. Santos, A. Abelha, J. Machado, A. Cabral, and I. Aragão. Electronic Health Records in the Emergency Room. ACIS-ICIS 2010, Aug. 2010.

[10] J. Reinschmidt and A. Françoise. Business Intelligence Certification Guide. International Technical Support Organization, Jan. 2000.

[11] A. Ribeiro. Implementação de um Sistema de Business Intelligence para Análise da Doença Pulmonar Obstrutiva Crónica. Master's thesis, Universidade do Minho, 2011.

[12] U. Varshney. Pervasive healthcare: Applications, challenges and wireless solutions. Communications of the Association for Information Systems, 16(3), 2005. 